\title{
Contraception chez les adolescentes
}

\author{
Margot A. Rosenthal MD, Sarah K. McQuillan MD
}

Citation : CMAJ 2021 August 9;193:E1218. doi : 10.1503/cmaj.202413-f

Voir la version anglaise de l'article ici : www.cmaj.ca/lookup/doi/10.1503/cmaj.202413

\section{Malgré une diminution de l'incidence des grossesses chez les adolescentes au \\ - Canada, la plupart d'entre elles sont non planifiées.}

Au Canada, les naissances vivantes chez les mères de moins de 19 ans représentaient 5,3\% des cas en 2000, $4 \%$ des cas en 2010 et $1,7 \%$ des cas en $2019^{1}$. Cependant, plus de $80 \%$ des grossesses de mères adolescentes sont non planifiées et plusieurs sont interrompues ${ }^{2}$. La Société canadienne de pédiatrie recommande un accès rapide aux méthodes de contraception et des ordonnances à renouvellement annuel afin de diminuer l'incidence des grossesses non planifiées et d'augmenter le taux de poursuite de la contraception ${ }^{3}$.

Les adolescentes font face à de nombreux obstacles pour accéder à des méthodes de contraception.

Plusieurs adolescentes sont inquiètes de leur capacité à payer pour des méthodes de contraception et mentionnent éprouver des difficultés à avoir accès à des soins confidentiels en la matière ${ }^{2}$. Les professionnels de la santé devraient insister sur les avantages de la confidentialité entre un médecin et sa patiente tout en expliquant les situations d'exception, y compris la nécessité de divulguer les comportements autodestructeurs et les activités se déroulant en situation de vulnérabilité.

Les méthodes contraceptives réversibles à longue durée d'action sont

recommandées pour les interventions de première ligne.

Parmi les populations où l'utilisation de méthodes contraceptives réversibles à longue durée d'action est élevée, le taux de grossesses chez les adolescentes est en diminution ${ }^{3}$. Les dispositifs intra-utérins libérant du lévonorgestrel et ceux à base de cuivre sont des exemples de ce type de méthodes efficaces disponibles au Canada ${ }^{3}$. En 2020, on a approuvé l'utilisation d'un implant libérant de l'étonogestrel (Naxplanon). Comme il est implanté dans la partie supérieure du bras et ne nécessite pas d'examen pelvien, cette option pourrait devenir populaire chez les adolescentes. Après une séance de consultation, la plupart des adolescentes choisissent une méthode contraceptive réversible à longue durée d'action lorsqu'elle est offerte gratuitement, avec un taux d'adoption comparable pour les implants et les dispositifs intra-utérins ${ }^{4}$.

Au cours de l'adolescence, la minéralisation des os représente jusqu'à $40 \%$ de l'accumulation de la masse osseuse observée à l'âge adulte ${ }^{5}$. Des études prospectives indiquent que les contraceptifs oraux contenant $\leq 30 \mu \mathrm{g}$ d'éthinylœstradiol peuvent être insuffisants pour optimiser la densité osseuse $e^{5}$. Les utilisateurs d'acétate de médroxyprogestérone (Depo-Provera) à long terme pourraient subir une diminution de leur densité minérale osseuse; l'interruption de la médication peut renverser la perte de densité 5 . Les implants et les dispositifs intra-utérins ne semblent pas affecter l'accumulation osseuse. 
5 Encouragez l'utilisation concomitante de méthodes barrières

de contraception afin de prévenir les infections transmissibles sexuellement.

Environ $50 \%$ de l'ensemble des infections transmissibles sexuellement se produisent au sein du groupe d'âge des 15-24 ans, mais les adolescentes qui emploient des méthodes contraceptives réversibles à longue durée d'action sont $60 \%$ moins susceptibles d'avoir recours au condom, comparativement à celles à qui on a prescrit des contraceptifs oraux ${ }^{6}$.

\section{Références}

1. Table 13-10-0416-01: Live births, by age of mother. Ottawa: Statistics Canada. Accessible ici : https://www150.statcan.gc.ca/t1/tbl1/en/tv.action?pid=1310041601 (consulté le 18 oct. 2020).

2. Di Meglio G, Yeates J, Seidman G. Can youth get the contraception they want? Results of a pilot study in the province of Quebec. Paediatr Child Health 2020;25:160-5.

3. Di Meglio G, Crowther C, Simms J. Contraceptive care for Canadian youth. Paediatr Child Health 2018;23:271-7.

4. Secura GM, Madden T, McNicholas C, et al. Provision of no-cost, long-acting contraception and teenage pregnancy. N Engl J Med 2014;371:1316-23.

5. Golden NH. Bones and birth control in adolescent girls. J Pediatr Adolesc Gynecol 2020;33:249-54.

6. Steiner RJ, Liddon N, Swartzendruber AL, et al. Long-acting reversible contraception and condom use among female US high school students: implications for sexually transmitted infection prevention. JAMA Pediatr 2016;170:428-34.

Intérêts concurrents : Aucun déclaré.

Cet article a été révisé par des pairs.

Affiliations : Département d'obstétrique, de gynécologie et des sciences de la reproduction (Rosenthal), Université du Manitoba, Winnipeg, Man.; Département d'obstétrique et de gynécologie (McQuillan), Université de Calgary, Calgary, Alb.

Propriété intellectuelle du contenu : Il s'agit d'un article en libre accès distribué conformément aux modalités de la licence
Creative Commons Attribution (CC BY-NCND 4.0), qui permet l'utilisation, la diffusion et la reproduction de tout médium à la condition que la publication originale soit adéquatement citée, que l'utilisation se fasse à des fins non commerciales (c.-àd., recherche ou éducation) et qu'aucune modification ni adaptation n'y soit apportée. Voir : https://creativecommons.org/ licenses/by-nc-nd/4.0/deed.fr.

Correspondance : Margot Rosenthal, umrose32@myumanitoba.ca 